\title{
Continuous Electroencephalography in the Medical ICU
}

\author{
Alejandro A. Rabinstein
}

Published online: 6 August 2009

(C) Humana Press Inc. 2009

When evaluating patients with coma or altered consciousness in non-neurological ICUs, I often wonder when I should be ordering electroencephalography (EEG). EEG is necessary to exclude non-convulsive status epilepticus but continuous EEG (cEEG) is required to assess for intermittent non-convulsive seizures. It is fairly clear that nonconvulsive epileptiform activity is relatively common in comatose critically ill neurological patients (such as patients with subarachnoid hemorrhage, intracerebral hematomas, and severe traumatic brain injury), and it is associated with worse outcomes in this population [1-3]. However, the incidence and impact of clinically occult epileptiform activity in critically ill patients without a primary brain insult has been less well defined.

A recently published study by Oddo et al. [4] from Columbia University contributes to fill this gap in our knowledge. The investigators reviewed the data of 201 consecutive patients who underwent cEEG monitoring in their medical ICU. Sepsis was the primary diagnosis in $60 \%$ of the cases and $48 \%$ of the patients were comatose at the time of initiation of cEEG. Over one in five patients had electrographic seizures (ESz, present in $10 \%$ ), periodic epileptiform discharges (PEDs, present in $17 \%$ and most often generalized), or both. These EEG abnormalities were not associated with any clinical manifestations (i.e., purely non-convulsive) in approximately two-thirds of cases. Sepsis was by far the most common diagnosis among patients with ESz or PEDs (84\%), and they were detected in almost one-third of septic patients monitored with cEEG. In

A. A. Rabinstein $(\square)$

Division of Critical Care Neurology, Department of Neurology,

W8B, Mayo Clinic College of Medicine, 200 First Street SW,

Rochester, MN 55905, USA

e-mail: rabinstein.alejandro@mayo.edu fact, sepsis was the only variable found to be independently associated with the occurrence of ESz and PEDs on cEEG (odds ratio [OR] 4.6, 95\% confidence intervals [CI] $1.9-12.7, P=0.002$ ). Poor outcome (mortality or severe dependency) upon hospital discharge was significantly more common among patients with ESz and PEDs, even after controlling for age, coma, and organ failures (adjusted OR $19.1,95 \%$ CI $6.3-74.6, P<0.001)$.

We learn from this study that ESz and PEDs are also relatively frequent in critical patients without known primary neurological injury, confirming findings from a previous cohort, which also included some neurological patients [5]. It also indicates that patients with these EEG abnormalities may have worse prognosis. However, several caveats need to be considered when interpreting the prognostic implications of this analysis. Sepsis was the only homogeneous diagnostic category considered in this study and diagnosis of sepsis was not included in the multivariable analysis evaluating predictors of outcome. Therefore, given the strong association between ESz and PEDs on cEEG and sepsis, it is possible that the association of these epileptiform abnormalities and poor outcome could be explained by the effects of sepsis. Also, the diagnosis of coma at the time of cEEG initiation was based on the exams described in the clinical records. As the level of neurological expertise of the examiners may have varied, one cannot be sure that cEEG is actually a more reliable predictor of poor outcome than the presence of coma, as it appears suggested by this study. Finally, the effect of withdrawal of life support (yes, the infamous self-fulfilling prophecy once again) may have influenced the association between cEEG findings and clinical outcome.

Hence, we know that cEEG may show non-convulsive ESz and PEDs in up to one-third of selected patients with sepsis-associated brain dysfunction. We now need to 
design prospective studies to determine the true incidence of non-convulsive ESz in encephalopathic septic patients at large, the influence of these EEG findings on functional prognosis (including functional outcome after hospital discharge and using more detailed assessment scales), the impact of antiepileptic treatment in these cases, and the cost-effectiveness of cEEG monitoring in the medical ICU.

\section{References}

1. Claassen J, Hirsch LJ, Frontera JA, Fernandez A, Schmidt M, Kapinos G, et al. Prognostic significance of continuous EEG monitoring in patients with poor-grade subarachnoid hemorrhage. Neurocrit Care. 2006;4(2):103-12.

2. Claassen J, Jetté N, Chum F, Green R, Schmidt M, Choi H, et al. Electrographic seizures and periodic discharges after intracerebral hemorrhage. Neurology. 2007;69(13):1356-65.

3. Vespa PM, Miller C, McArthur D, Eliseo M, Etchepare M, Hirt D, et al. Nonconvulsive electrographic seizures after traumatic brain injury result in a delayed, prolonged increase in intracranial pressure and metabolic crisis. Crit Care Med. 2007;35(12):2830-6.

4. Oddo M, Carrera E, Claassen J, Mayer SA, Hirsch LJ. Continuous electroencephalography in the medical intensive care unit. Crit Care Med. 2009;37(6):2051-6.

5. Towne AR, Waterhouse EJ, Boggs JG, Garnett LK, Brown AJ, Smith JR Jr, et al. Prevalence of nonconvulsive status epilepticus in comatose patients. Neurology. 2000;54(2):340-5. 